THE

\title{
RESULTS IN THIRTYY-NINE CASES
}

\author{
OF \\ EX C I I O N OF TH E K N E. \\ BY \\ GEORGE MURRAY HUMPHRY, M.D., F.R.S., \\ PROFESSOR OF ANATOMY, AND SURGHON TO $\triangle$ DDENBROOKE'S HOSPITAL, \\ CAMBRIDGF.
}

Received Oct. 14th.-Read Nov. 10th, 1868.

THE following may be regarded as supplementary to a paper communicated by me to this Society and published in Vol. XLI of its 'Transactions.' The first thirteen of the cases are related there, and observations are offered respecting the cases most suited to the operation, \&c., to which I will merely refer inasmuch as they have been, in almost every particular, fully confirmed by my subsequent experience.

In 39 cases in which $I$ have performed the operation of excision of the knee, the result has been as follows :

28 recovered. 2 died. 9 underwent amputation; of these 5 recovered and 4 died. ${ }^{1}$

All the cases were treated in Addenbrooke's Hospital.

1 Since this paper was read I have performed the operation six times; five of the patients have recovered, and one is under treatment. This makes the number of operations 45, and the number of recoveries 33.-July, 1869. 
Of the two patients who died without amputation, one (No. 8) was the girl, æt. 5, whose case is related at p. 203 of the volume just referred to. The operation was performed, perhaps rather unwisely, on account of acute suppuration in the joint, supervening upon chronic disease with an opening into the joint, and attended with violent fever. The condition was most unfavorable for any operation, and the child would probably have died whatever course was taken. In the other (a female, æt. 25, No. 31) death was caused by a violent attack of hæmatemesis commencing a few days after the operation, and having, apparently, no particular relation to it.

Of the four who died after amputation the excision was in one (No. 29) performed in consequence of an extensive contused wound of the thigh, caused by a wheel passing over it, laying open the knee-joint and admitting a quantity of gravel and dirt into it. The lad was restless and delirious after the operation, with slight discharge from the wound, but there was no swelling or other indication of inflammation. As he became worse, I amputated on the fourth day. The restlessness continued, mortification of the stump supervened, and he died three days after the amputation.

In the other three cases amputation was performed in consequence of continued suppuration undermining the health, and was, in each instance, delayed too long.

In the remaining five cases in which amputation was performed, the reason for the removal of the limb was the same as in the three last mentioned. In one case, at least, I thought the extensive and continued suppuration was caused by bleeding taking place into the wound after the patient was replaced in bed, and I have accordingly been very careful in the later cases to prevent this occurrence by securing all the vessels that were at all likely to bleed. In two, if not three, of the cases I judged, from examination of the part after amputation, that the limb might have been saved if $I$ had made incisions in the course of the discharging sinuses, so as to expose or remove the carious edges of the bones.

In each of the cases of recovery the cut surfaces of 
the tibia and femur united together, and a good, firm, sound limb was gained. In four, in consequence either of an accident or of the patient's bearing upon the limb before firm union of the bones had taken place, the part became more bent than is desirable; in one of these (No. 15) I subsequently straightened the limb by removing a V-shaped piece from the forepart of the angle, and the patient has now, I am told, a straight limb, and can walk well. The other three are too satisfied with their condition to assent to any further operation. One, with the aid of a wood and iron addition to his limb, says "he can walk and work with most of them." Another considers the flexure rather advantageous, and says he can walk and work as well as other people. The third patient also can walk and work very fairly.

Through the kindness of medical men, and in other ways, $I$ have been able to obtain information respecting the condition of most of the patients at various periods since the operation, and the result is highly satisfactory. The limb is, of course, stiff at the knee, but there is no other inconvenience. The slight shortening caused by the removal of the articular surfaces is no disadvantage. On the contrary, it facilitates the swing of the foot forwards in walking and running. There has been no tendency to return of disease at the part in any that $I$ have heard of.

In the majority of the cases the disease in consequence of which the operation was performed had commenced in the synovial membrane, and had made its way slowly through the cartilages, more or less deeply, into the bones, so as to leave little or no prospect of a useful joint or of the limb being preserved.

In some the disease had passed away and sinuses resulting from an ulcerated state of the bones had healed, but the joint was contracted and the articular surfaces were distorted beyond the hope of benefit from extension and mechanical appliance. In these two classes of cases, and in some others, excision offers the prospect of a very useful limb, a better prospect, so far as my experience goes, than does any other 
procedure, and it does so with little risk. I say with little risk, because the operation, though extensive, is, as in the case of other operations in which osseous structures are chiefly concerned, not usually followed by much constitutional disturbance. No large vessels or nerves, and not much muscular tissue, are involved. The chief danger to be feared is a continuance of suppuration undermining the health and rendering amputation necessary. This may probably be lessened by improved treatment after the operation. It is most likely to occur in patients of strumous temperament. In such persons, as mentioned in my former paper, disease lingers long in the synovial membrane, causing thickening and other changes in it, and tedious suppuration is not unlikely to follow excision. This was the most frequent cause of failure in my cases.

There is no longer any doubt that the growth of a limb after excision of the knee, in a young subject, may keep pace with that of the opposite limb, provided the epiphysial or growing lines at the end of the femur and the tibia have not been removed or injured, and provided other circumstances are favorable. This is shown by Case 30 and others. Nevertheless, just as growth is commonly more or less impaired when muscular action in a limb is prevented by paralysis, by disease, or anchylosis of a joint, so some years after excision it is not unfrequently found that the limb is shorter than its fellow, to an amount which is scarcely to be accounted for by the loss of bone incurred in the operation. Thus, in Case 13, ten years after the operation, performed at the age of thirteen, the operated femur measured sixteen inches and the tibia fourteen; these bones in the opposite limb measuring respectively eighteen inches and fourteen and a half. The operated tibia had grown as fast as the other; but, for some reason not quite obvious, the operated femur had been less active in its growth than its uninjured compeer. In Case 22, six years after the operation, performed at the age of fourteen, the femur and tibia were each two inches shorter in the operated than in the other limb. In Case 15, three years after the operation, performed at the 
age of ten, the operated femur measured thirteen inches and the tibia ten and three quarters; in the opposite limb the femur measured fourteen inches and the tibia eleven and three quarters. It may be remarked that in each of these cases the limb had become bent after the operation, and it is quite probable that anchylosis of the two bones in this position may be more unfavorable to their growth than if they had remained straight.

It is, however, important to observe that the limb, whether it remained short or grew in conformity with the other, whether it continued straight or became bent, was in every instance a very useful limb; and it was so because all disease had been cleared away. The weakness associated with disease and impaired structure was gone, and a firm sound limb attained, such as it can rarely become so long as the altered articular surfaces remain. It is upon this complete removal of the morbid and weakened tissues and the union of the clean cut osseous surfaces of the bones, forming a solid weightbearing column, that the advantages of excision of such a joint as the knee mainly depend.

The operation of excision of the knee recommends itself, therefore, partly at least, on the following grounds. When disease has proceeded to such an extent as seriously to alter or destroy the synovial, cartilaginous, or osseous structures of the joint (which, be it observed, commonly may, and in the upper class of society usually is, prevented by early attention and rest), it is almost in vain to expect that a useful joint can be restored, by which I mean a joint with useful movement and able to bear the weight of the body well. Indeed, the limited movement which remains is, commonly, worse than useless; it is mischievous, by entailing weakness, with liability to return of disease, and permitting contraction to ensue. The best result that can take place in such a case is the complete abolition of the joint with anchylosis of the bones. Even if the limb be bent or otherwise distorted, it is more likely to be useful when anchylosed than if movement continued. Unfortunately, as we know, this result does not very often take place at the knee. As the disease subsides vol. L1I. 
the articular surfaces become united, and that, perhaps, in parts only, by a soft fibrous medium, which permits some movement, and has little disposition to ossification even under the long-continued favorable condition of perfect rest. In most of the limbs rendered crippled, useless, and wasted by disease of the knee, some movement of the joint remains, insufficient for any useful purpose, but enough to disable the limb from bearing weight; and mechanical supplements, even if they answer their purpose, are by most persons unattainable. By excision this difficulty is overcome, the remaining portions of the joint, which serve only to render the limb useless, are removed, and anchylosis of the bones is effected. In short, we bring about by the operation that condition which we would gladly see attained without such interference.

I usually commence the operation by a semilunar incision, with the convexity downwards in front of the knee, then reflect the skin a little, cut into the joint, remove the patella and as much of the diseased synovial membrane as I can, expose the articular surfaces, and saw off the diseased parts.

It is sometimes necessary to saw off a second or a third slice, or to gouge out ulcerated or diseased portions ; and it is better to apply the saw two or three times than to remove recklessly large portions at first, though this may have the trifling advantage of expediting the procedure. I do not reflect the circa-periosteal tissues close, but prefer to saw them off with the bones, so as to leave the latter well covered at their edges, and therefore less liable to ulcerate. It is also very important so to saw the bones that when the cut surfaces of the tibia and femur are placed in contact they fit well to each other, the line of the limb being straight, $i$. $e$. not bent or with the leg inclined to either side.

I am very careful to sponge the wound well and secure the bleeding vessels, so as to prevent hæmorrhage taking place after the patient is placed in bed, for the collection of blood in the wound prevents early union and entails suppuration. Ligatures should, if possible, be avoided, and torsion used instead, so that no foreign body is left in the wound. 
Having taken these precautions and placed the cut surfaces of the bones in as complete apposition as possible, I bandage the limb along its whole length, except in the line of the wound, upon a splint placed behind and provided with a foot-piece. I then unite the edges of the wound by sutures and apply long splints on the sides, with pads placed above and below the knee, so as to defend the operated part from undue pressure, and to permit discharge to escape in the event of suppuration taking place. The limb is thus firmly secured in its whole length, except at the wound, which is left exposed. The bandages are moistened with blood, which sets and affords additional security. I am very particular to adjust things well and firmly in the first instance, and am very unwilling to disturb the limb afterwards. Indeed, I frequently do not remove any of the bandages or splints for five, six, or more weeks; and by this care and perfect quiet I endeavour to promote immediate union, which, not uncommonly, takes place throughout the entire wound. If this fails to take place and suppuration ensues, I still do not remove or change the bandages and splints, unless there is some unusual reason for doing so, but allow the discharge to escape at the sides and adopt various means to maintain cleanliness. This perfect quietude and abstinence from removal of the bandages first applied is, I consider, a very important item in the treatment. It is equally important not to discontinue the splints till the bones are quite firmly united, till the patient can raise the limb from the bed by its own muscles, without any movement being perceptible between the tibia and femur. It is necessary to make quite sure of this. For that purpose I watch closely when the effort to raise the limb from the bed is first made, because any slight movement is then most easily detected; and if the patient is allowed to get up while movement, however slight, takes place between the bones, the part is liable to become bent, or the process of anchylosis may be suspended, and the good result is thus marred. This flexure, as already stated, took place in some of my cases. It, as well as failure of firm union between the bones, has occurred in the practice of 
others; but they may be always, or generally, avoided by attention to the directions I have given.

The cancellous parts of bones, whether broken or cut, commonly unite well and quickly, and the opposed surfaces of the tibia and femur, after excision of the knee, form no exception to this rule. I have often been surprised at the rapidity with which the wound healed and the bones grew together.

When suppuration takes place and continues, careful management is required, for which it is impossible to lay down rules. Much judgment also is needed in deciding on the propriety of laying open sinuses and exposing or removing carious bone, and still more in determining the period beyond which it is unsafe to defer the removal of the limb. The result proved in some of my cases that $I$ had delayed this too long; and in others the examination of the part after amputation indicated that, with additional patience and welldirected incisions, the limb might have been spared.

Excision of the knee is an operation requiring care in the execution, and care and patience in the subsequent management; but it is not an operation of much danger, and by it, I am sure, many an amputation might be avoided and many crippled, worse than useless, limbs, such as we daily see carried about our streets, might be rendered good helpmates to their fellows. 


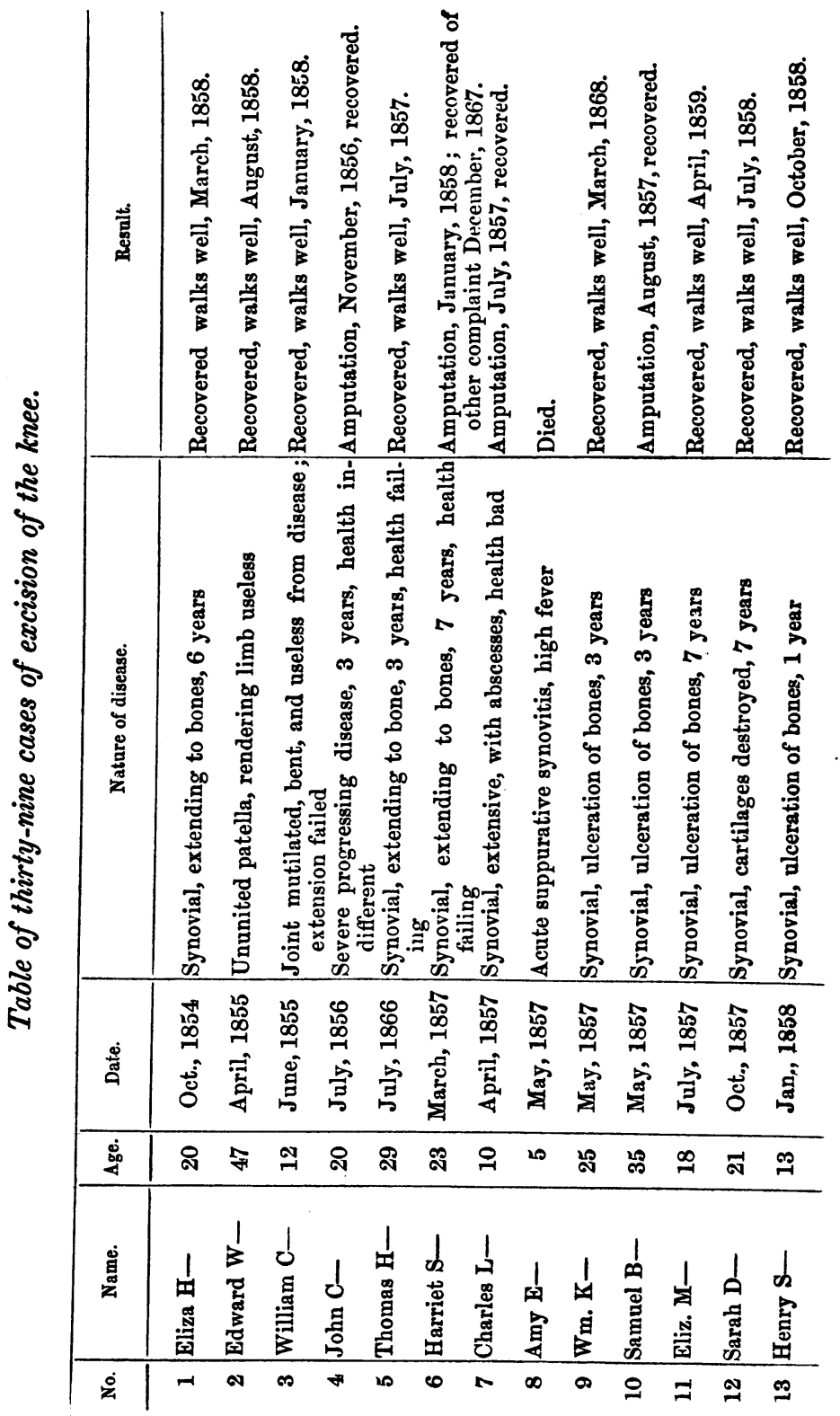




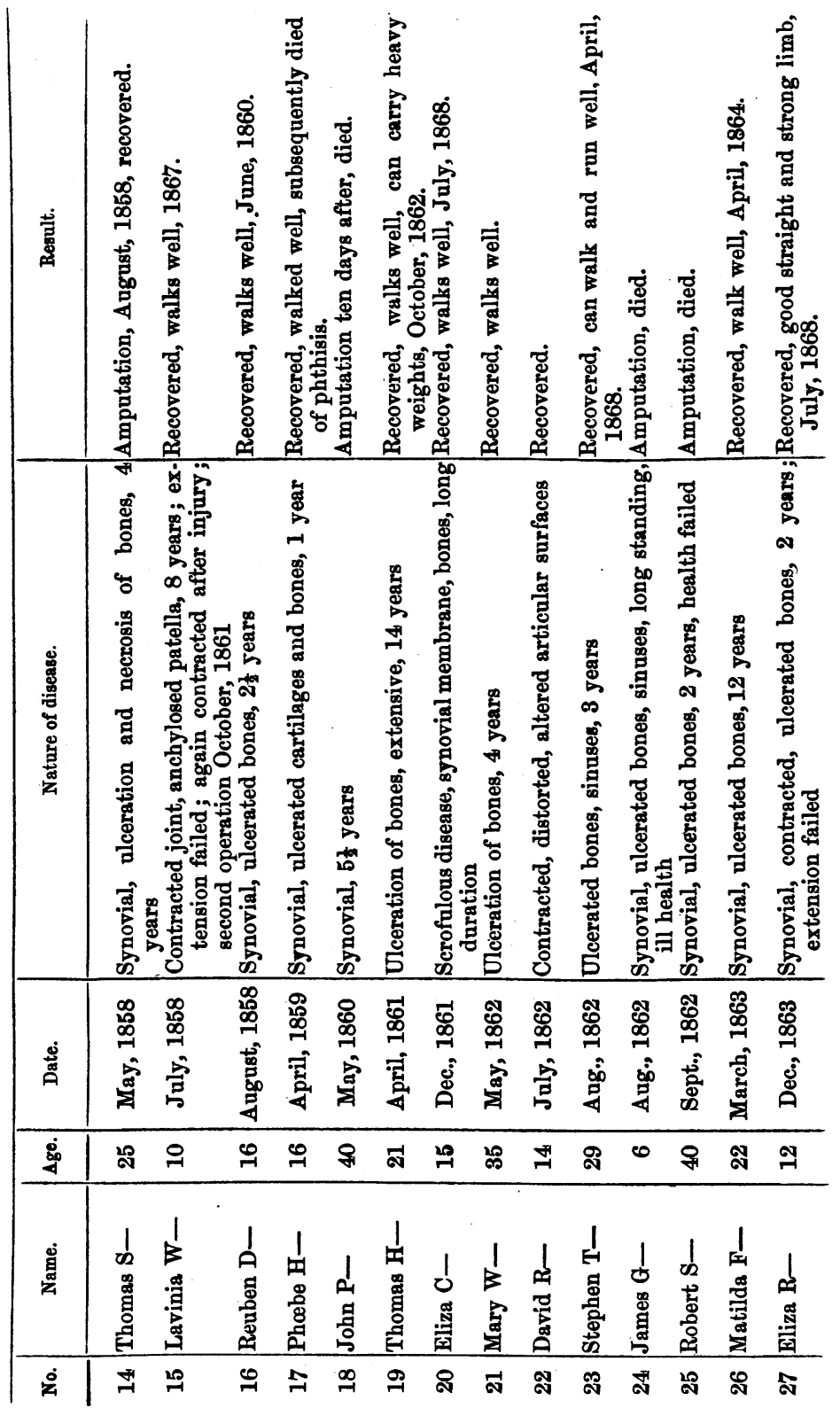


EXCISION OF THE KNEE:

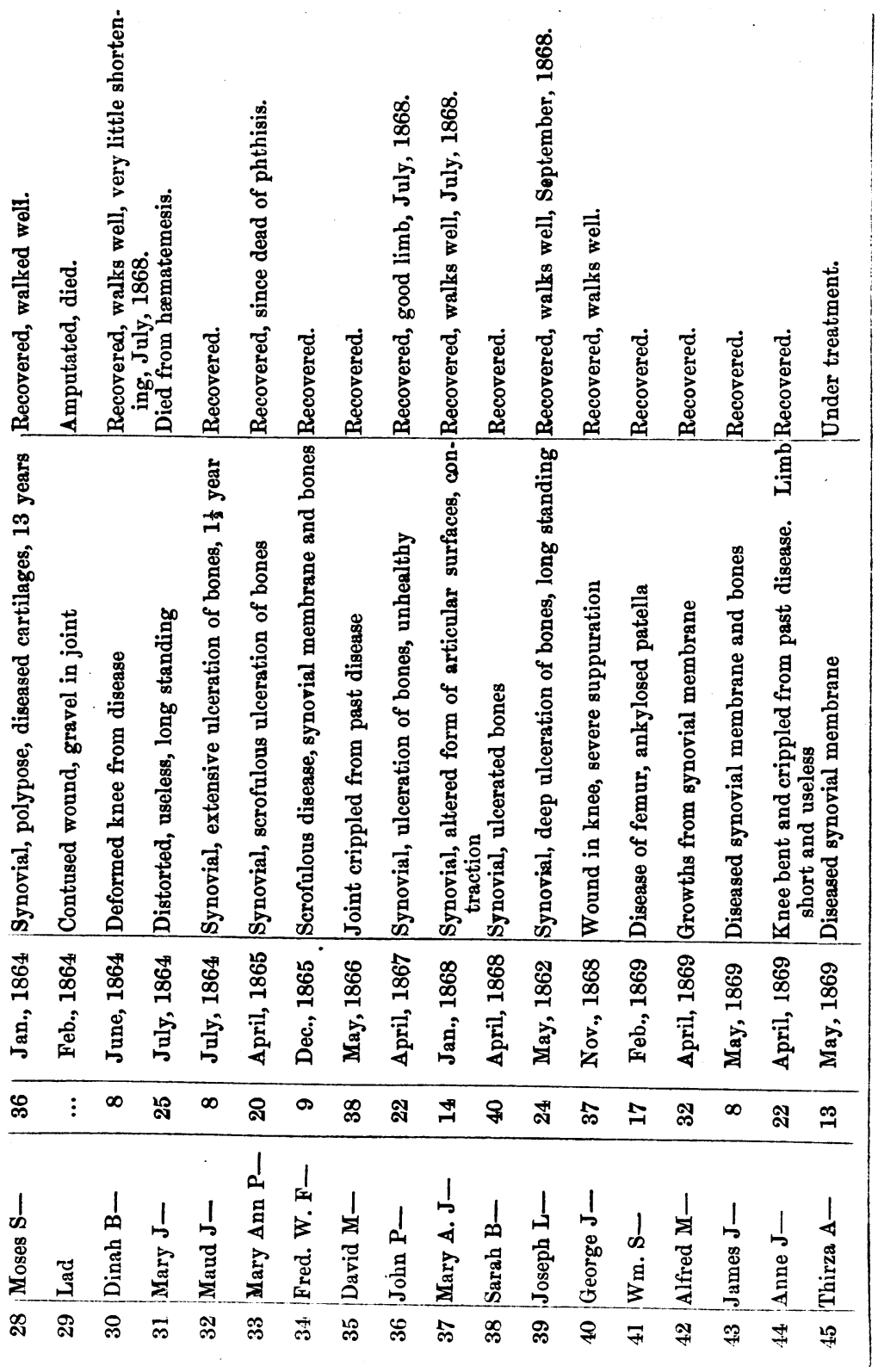


4. The amputation was performed in consequence of continuing suppuration, causing the health to fail. Portions of synovial membrane remained, and there was an absence of osseous union between the bones.

6. There was firm bony union; sinuses led down to carious bone. I advised the laying open these and removal of diseased bone, and regretted that this had not been tried, believing that the limb might have been saved.

7. Abscesses continued and health failed. Fibrous union of bones, no caries.

10. Was of restless irritable temperament and health failed, though there was nothing in limb to forbid recovery.

13. July, 1868.-The knee is bent, which he considers rather an advantage. The right femur measures sixteen inches, the left eighteen. The right tibia measures fourteen inches, the left fourteen and a half.

14. A good deal of bleeding after the operation into the wound, causing suppuration, was, I thought, one cause of this case doing badly. The bones were united by fibrous structure, edges carious. Free incisions and removal of carious edges might, probably, have saved this limb.

15. Before firm union had taken place she hurt the limb. It became bent, so that she went lame, and I accordingly performed the operation a second time, October, 1861.

22. Was beginning to walk well upon the limb when he was thrown from a horse and the limb bent under him. It remained bent, and is now, July, 1868, bent at an angle of about $100^{\circ}$. The bones are firmly united, but the tibia and femur are each two inches shorter than in the other leg. He walks upon the limb, though lamely.

24. Suppuration went on, weakening the child. Amputation delayed too long.

25. Profuse suppuration, lowering the man, so that he sank after amputation, which was delayed too long. 
29. The tissues about the joint lacerated, contused, and ingrained with gravel from wheel of loaded waggon passing over; restless, delirious; dirty discharge. Amputation on fourth day. Continued restlessness till death.

31. Was going on well when violent hæmatemesis occurred and proved fatal. She had long been subject to flatulence and pain after food. 